\title{
Some Oscillation Criteria for Higher-Order Neutral Emden-Fowler Delay Dynamic Equations on Time Scales
}

Ying Sui, Yulong Shi, Yibin Sun and Shurong Sun ${ }^{a}$

College of Sciencel, University of Jinan, Jinan, Shadong 250000, P R China

\begin{abstract}
New oscillation criteria are established for higher-order Emdn-Fowler dynamic equation $q(v) x^{\beta}(\delta(v))+\left(r(v)\left(z^{\Delta^{n-1}}(v)\right)^{\alpha}\right)^{\Delta}=0$ on time scales, $z(v):=p(v) x(\tau(v))+x(v)$. Our results extend and supplement those reported in literatures in the sense that we study a more generalized neutral delay equation and do not require $r^{\Delta}(v) \geq 0$ and the commutativity of the jump and delay operators.
\end{abstract}

\section{Introduction}

We will consider the even order dynamic equation

$$
q(v) x^{\beta}(\delta(v))+\left(\left(z^{\Delta^{n-1}}(v)\right)^{\alpha} r(v)\right)^{\Delta}(v)=0, t \in V,
$$

where $z(v):=p(v) x(\tau(v))+x(v), V=\left[t_{0}, \infty\right)_{T}$, and $n$ is an even integer, $n \geq 2, \alpha>0, \beta>0$ are quotient of odd integers.

Assume that:

(A1) $p(v), r(v), q(v) \in C_{r d}(V,(0, \infty))$, where $p(v)<1$ and $\int_{v_{0}}^{\infty} \frac{1}{r^{\frac{1}{\alpha}}(s)} \Delta s=\infty$.

(A2) $\tau(v), \delta(v) \in C_{r d}(V, T)$, such that $v \geq \tau(v)$, $v \geq \delta(v)$, and $\lim _{v \rightarrow \infty} \tau(v)=\lim _{v \rightarrow \infty} \delta(v)=\infty$.

Grace [1] studied an even order equation

$$
q(v)(x(v))^{\alpha}+\left(\left(x^{\Delta^{n-1}}(v)\right)^{\alpha} a(v)\right)^{\Delta}=0,
$$

where $n \geq 2, \alpha \geq 1$ is the ratio of positive odd integers. Under the conditions $a^{\Delta}(t) \geq 0$ and $\int^{\infty} \frac{1}{a^{\frac{1}{\alpha}}(s)} \Delta s=\infty$. some oscillation criteria of (2) were established.

Tunç [2] considered the oscillatory of even order equation

$$
\begin{aligned}
& F\left(v, x(v), x(\tau(v)), x^{\Delta}(v), x^{\Delta}(\tau(v))\right) \\
& +\left(x^{\Delta^{n-1}}(v)\left|x^{\Delta^{n-1}}(v)\right|^{\alpha-1} r(v)\right)^{\Delta}=0,
\end{aligned}
$$

where $n \geq 2, \alpha>0$ is a constant and exists a rd-continuous function $q(v)>0$ such that

$$
\frac{F(v, x, u, i, w)}{|u|^{\alpha-1} u} \geq q(v) .
$$

Furthermore, assume $\tau(v)$ satisfy $\tau^{\Delta}(v)>0$, $\tau(\sigma(v))=\sigma(\tau(v))$. By using Taylor monomials, some sufficient conditions for ensuring (3) oscillatory are established.

On the basis research of Grace [1] and Tunç [2], we study a neutral delay dynamic equation (1), by Ricati transformation with inequality technique and using comparison principles, sufficient conditions for oscillation of (1) extend and supplement some known results, and allow to relax some restrictive conditions imposed on the equation, like $r^{\Delta}(v) \geq 0$.

\section{Auxiliary lemmas}

We use the polynomials $\left\{h_{k}(v, s)\right\}_{k=0}^{\infty}$ (see [2]), where

$$
\eta_{i}(v, s)= \begin{cases}\int_{s}^{v} \eta_{i-1}(u, s) \Delta u, & i \in N \\ 1, & i=0\end{cases}
$$

Lemma 1. (Taylor's formula [3]). If $f \in C_{r d}^{n}(T, R)$, $n \in N$. For $s \in T$

$$
\begin{aligned}
f(v)= & \int_{s}^{\rho^{n-1}(v)} f^{\Delta^{n}}(\eta) h_{n-1}(v, \sigma(\eta)) \Delta \eta \\
& +\sum_{k=0}^{n-1} f^{\Delta^{k}}(s) h_{k}(v, s), v \in T
\end{aligned}
$$

Lemma 2. [4]. If $f \in C_{r d}(T, R), n \in N$, for $s, v \in T$,

\footnotetext{
a Corresponding author: sshrong@163.com
} 


$$
\begin{aligned}
& \int_{s}^{v} f(\eta) h_{n}(s, \sigma(\eta)) \Delta \eta(-1)^{n} \\
& =\int_{s}^{v} \int_{\eta_{n+1}}^{v} \cdots \int_{\eta_{2}}^{t} f\left(\eta_{1}\right) \Delta \eta_{1} \Delta \eta_{2} \cdots \Delta \eta_{n+1}
\end{aligned}
$$

Lemma 3. [5]. If $\sup T=\infty, f \in C_{r d}^{n}(V, R)$, $n \in N$, then

(i) $\liminf f^{\Delta^{n}}(v)>0$

Then $\lim _{v \rightarrow \infty} f^{\Delta^{i}}(v)=\infty, \quad i \in[0, n)_{Z}$.

(ii) $\limsup f^{\Delta^{\prime \prime}}(v)<0$

Then $\lim _{v \rightarrow \infty} f^{\Delta i}(v)=-\infty, i \in[0, n)_{Z}$.

Lemma 4. (Kiguradz's lemma [5]). Let $\sup T=\infty$, $f \in C_{r d}^{n}\left(V, R^{+}\right), \quad n \in N$. Suppose that either $f^{\Delta^{n}} \leq 0(\equiv 0)$ or $f^{\Delta^{n}} \geq 0(\not \equiv 0),\left[v_{0}, \infty\right)_{T}$. Then, $f^{\Delta^{n}}(v)(-1)^{n-m} \geq 0 \quad, \quad m \in[0, n]_{Z} \quad v \in\left[v_{1}, \infty\right)_{T} \quad$, $v_{1} \in\left[v_{0}, \infty\right)_{T}$,

(i) $f^{\Delta i}(v)>0, \quad i \in[0, m)_{Z}, v \in\left[v_{1}, \infty\right)_{T}$.

(ii) $f^{\Delta^{i}}(v)(-1)^{a+i}>0, i \in[a, n)_{Z}, v \in\left[v_{1}, \infty\right)_{T}$.

Lemma 5. [3] $x$ is differentiable,

$\left(g^{n}\right)^{\Delta}(v)=n g^{\Delta}(v) \int_{0}^{1}\left[h g^{\sigma}(v)+(1-h) g(v)\right]^{n-1} d h$,

$n$ is constant.

Lemma 6. Assume that (A1)-(A2), and $x(v)>0$ is an solution of (1). If

$$
\int_{v_{0}}^{\infty} r^{-\frac{1}{\alpha}}(v) h_{1}\left(\sigma(w), v_{0}\right)\left(\int_{w}^{\infty} Q(s) \Delta s\right)^{\frac{1}{\alpha}} \Delta w=\infty,
$$

where $(-p(\delta(v))+1)^{\beta} q(v)=Q(v)$.

For $v_{1} \in\left[v_{0}, \infty\right)_{T}$ $z^{\Delta^{k}}(v)>0,(k=0,1, \cdots, n-1)$,

$$
\left(\left(z^{\Delta^{n-1}}(v)\right)^{\alpha} r(v)\right)^{\Delta}<0 .
$$

Proof. If $x(t)>0$ is solution of (1), then $x(\tau(v))>0$ and $x(\delta(v))>0$ for $v \in\left[v_{1}, \infty\right)_{T}, \quad v_{1} \in\left[v_{0}, \infty\right)_{T}$ then $z(v)>0$. From (1) and (A1),

$$
-x^{\beta}(\delta(v)) q(v)=\left(\left(z^{\Delta^{n-1}}(v)\right)^{\alpha} r(v)\right)^{\Delta}<0,
$$

thus $z^{\Delta^{n-1}}(v)$ is eventually of one sign.

We claim that

$$
z^{\Delta^{n-1}}(v)>0, v \in\left[v_{1}, \infty\right)_{T} .
$$

Not, $z^{\Delta^{n-1}}(v)<0$ on $\left[v_{2}, \infty\right)_{T}, v_{2} \geq v_{1}$. In view of (6), for $c>0, v \in\left[v_{2}, \infty\right)_{T}$

$$
\left(z^{\Delta^{n-1}}(v)\right)^{\alpha} r(v) \leq\left(z^{\Delta^{n-1}}\left(v_{2}\right)\right)^{\alpha} r\left(v_{2}\right)=-c .
$$

Thus,

$$
z^{\Delta^{n-1}}(v) \leq-\left(\frac{c}{r(v)}\right)^{\frac{1}{\alpha}}, v \in\left[v_{2}, \infty\right)_{T} .
$$

Then

$$
z^{\Delta^{n-2}}(v) \leq-\int_{v_{2}}^{v}\left(\frac{1}{r(s)}\right)^{\frac{1}{\alpha}} c^{\frac{1}{\alpha}} \Delta s+z^{\Delta^{n-2}}\left(v_{2}\right), v \in\left[v_{2}, \infty\right)_{T} .
$$

Letting $\quad v \rightarrow \infty \quad$ and from (A1), thus $\lim _{v \rightarrow \infty} z^{\Delta^{n-2}}(v)=-\infty$. From Lemma 3, $\lim _{v \rightarrow \infty} z(v)=-\infty$. This contradicts $z(v)>0$.

From Lemma 4, there exists $m \in[0, n-1]_{Z}$ such that $0 \leq z^{\Delta^{n-1}}(v)(-1)^{n-1-m}$, so $m \in\{1,3, \cdots, n-1\}$ such that (i) and (ii) of Lemma 4 hold, $v \in\left[v_{1}, \infty\right)_{T}, z^{\Delta}(v)>0$ on $v \in\left[v_{1}, \infty\right)_{T}$. Then, from (A1) and $z(v)$, we have

$$
\begin{aligned}
x(v) & =-p(v) x(\tau(v))+z(v) \\
& \geq-p(v) z(\tau(v))+z(v) \\
& \geq(-p(v)+1) z(v) .
\end{aligned}
$$

From (1),

$$
0 \geq z^{\beta}(\delta(v)) Q(v)+\left(\left(z^{\Delta^{n-1}}(v)\right)^{\alpha} r(v)\right)^{\Delta} .
$$

Now, we claim that $m=n-1$. If not, we have

$$
0<z^{\Delta^{n-3}}(v), 0>z^{\Delta^{n-2}}(v), v \in\left[v_{1}, \infty\right)_{T} .
$$

Since $\lim _{v \rightarrow \infty} \delta(v)=\infty$ and $z^{\Delta}(v)<0,\left[v_{1}, \infty\right)_{T}$, we can choose $v_{2} \in\left[v_{1}, \infty\right)_{T}, v_{1} \leq \delta(v), v \in\left[v_{2}, \infty\right)_{T}$, and so

$$
0<c=z\left(v_{1}\right) \leq z(\delta(v)), v \in\left[v_{2}, \infty\right)_{T} .
$$

Integrating (8) $v$ to $u \geq v, u \rightarrow \infty$, by (9),

$$
\left(\int_{t}^{\infty} Q(s) \Delta s \frac{1}{r(v)}\right)^{\frac{1}{\alpha}} c^{\frac{\beta}{\alpha}} \leq z^{\Delta^{n-1}}(v), v \in\left[v_{2}, \infty\right)_{T} .
$$

Integrating (10) $v$ to $w \geq t, w \rightarrow \infty$, then

$$
-\int_{v}^{\infty}\left(\int_{u}^{\infty} Q(s) \Delta s \frac{1}{r(u)}\right)^{\frac{1}{\alpha}} \Delta u \times c^{\frac{\beta}{\alpha}} \geq z^{\Delta^{n-2}}(v) .
$$

Integrating $v_{2}$ to $v$, and by Lemma 1 , 


$$
\begin{aligned}
& z^{\Delta^{n-3}}\left(t_{2}\right) \geq c^{\frac{\beta}{\alpha}} \int_{v_{2}}^{v} \int_{w}^{\infty}\left(\int_{u}^{\infty} Q(s) \frac{1}{r(u)} \Delta s\right)^{\frac{1}{\alpha}} \Delta u \Delta v \\
& =-c^{\frac{\beta}{\alpha}} \int_{v_{2}}^{v} h_{1}\left(v_{2}, \sigma(w)\right)\left(\int_{w}^{\infty} Q(s) \Delta s \frac{1}{r(w)}\right)^{\frac{1}{\alpha}} \Delta w \\
& =c^{\frac{\beta}{\alpha}} \int_{v_{2}}^{v} h_{1}\left(\sigma(w), v_{2}\right)\left(\int_{w}^{\infty} Q(s) \Delta s \frac{1}{r(w)}\right)^{\frac{1}{\alpha}} \Delta w,
\end{aligned}
$$

Which contradicts (4). Therefore, $m=n-1$, and from Lemma 4, we have

$$
\begin{aligned}
& z(v)>0, \\
& z^{\Delta}(v)>0, \\
& \cdots, \\
& z^{\Delta^{n-1}}(v)>0, \quad v \in\left[v_{1}, \infty\right)_{T} .
\end{aligned}
$$

This completes the proof.

Lemma 7. Let $n>2$. Assume that $x(v)$ is an eventually positive solution of (1), and satisfy the conditions of Lemma 6 . Then $z^{\Delta^{n-2}}(v)\left(\int_{v_{1}}^{v} \frac{1}{r^{1 / \alpha}(s)} \Delta s\right)^{-1}$ is non-increasing on $\left[v_{1}, \infty\right)_{T}$, and

$$
z(v)
$$

$$
\geq \frac{z^{\Delta^{n-2}}(v)}{\int_{v_{1}}^{\rho^{n-3}(v)} \frac{1}{r^{1 / \alpha}(s)} \Delta s} \int_{v_{1}}^{v}\left(h_{n-3}(v, \sigma(\eta)) \int_{v_{1}}^{\eta} \frac{1}{r^{1 / \alpha}(s)} \Delta s\right) \Delta \eta .
$$

Proof. By Lemma 6 we get (5), then

$$
\left[\left(z^{\Delta^{n-1}}(v)\right)^{\alpha} r(v)\right]^{\Delta}<0,\left[v_{1}, \infty\right)_{T},
$$

and

$$
\begin{aligned}
& -z^{\Delta^{n-2}}\left(v_{1}\right)+z^{\Delta^{n-2}}(v)=\int_{v_{1}}^{v} \frac{\left(r(s)\left(z^{\Delta^{n-1}}(s)\right)^{\alpha}\right)^{\frac{1}{\alpha}}}{r^{1 / \alpha}(s)} \Delta s \\
& \geq \int_{v_{1}}^{v} \frac{1}{r^{1 / \alpha}(s)} \Delta s \times\left(\left(z^{\Delta^{n-1}}(v)\right)^{\alpha} r(v)\right)^{\frac{1}{\alpha}}
\end{aligned}
$$

then

$$
\int_{v_{1}}^{v} r^{-\frac{1}{\alpha}}(s) \Delta s \times\left(\left(z^{\Delta^{n-1}}(v)\right)^{\alpha} r(v)\right)^{\frac{1}{\alpha}} \leq z^{\Delta^{n-2}}(v) .
$$

Thus, we can obtain

$$
\begin{aligned}
& \left(\left(\int_{v_{1}}^{v} \frac{1}{r^{\gamma / \alpha}(s)} \Delta s\right)^{-1} z^{\Delta^{n-2}}(v)\right)^{\Delta} \\
= & \int_{v_{1}}^{v} \frac{1}{r^{1 / \alpha}(s)} \Delta s \times z^{\Delta^{n-1}}(v)-z^{\Delta^{n-2}}(v) r^{-1 / \alpha}(v) \\
& \left(\int_{v_{1}}^{\sigma(v)} r^{-1 / \alpha}(s) \Delta s \int_{v_{1}}^{v} r^{-1 / \alpha}(s) \Delta s\right)^{-1} \\
\leq & 0 .
\end{aligned}
$$

So $z^{\Delta^{n-2}}(v)\left(\int_{v_{1}}^{v} r^{-1 / \alpha}(s) \Delta s\right)^{-1}$ is non-increasing on $\left[v_{1}, \infty\right)_{T}$. Next, using Lemma 1 ,

$z(v)$

$$
\begin{aligned}
= & \int_{v_{1}}^{\rho^{n-3}(v)} h_{n-3}(v, \sigma(\eta)) z^{\Delta^{n-2}}(\eta) \Delta \eta+\sum_{k=0}^{n-3} h_{k}(v, s) z^{\Delta^{k}}(s) \\
\geq & \int_{v_{1}}^{\rho^{n-3}(t)} z^{\Delta^{n-2}}(\eta) h_{n-3}(v, \sigma(\eta)) \Delta \eta \\
= & \int_{v_{1}}^{\rho^{n-3}(v)}\left(\int_{v_{1}}^{\eta} \frac{1}{r^{1 / \alpha}(s)} \Delta s \frac{z^{\Delta^{n-2}}(\eta)}{\int_{v_{1}}^{\eta} \frac{1}{r^{1 / \alpha}(s)} \Delta s} h_{n-3}(v, \sigma(\eta))\right) \Delta \eta \\
\geq & \left(\int_{v_{1}}^{v} r^{-1 / \alpha}(s) \Delta s\right)^{-1} z^{\Delta^{n-2}}(v) \\
& \int_{v_{1}}^{\rho^{n-3}(v)}\left(h_{n-3}(v, \sigma(\eta)) \int_{v_{1}}^{\eta} \frac{1}{r^{1 / \alpha}(s)} \Delta s\right) \Delta \eta
\end{aligned}
$$

This completes the proof.

\section{Main results}

Let

$$
\begin{gathered}
A(v)=\int_{v_{0}}^{v} r^{-\frac{1}{\alpha}}(s) \Delta s, I_{1}(v)=A(v)-A\left(v_{1}\right), \\
I_{i}(v)=\int_{v_{1}}^{v} I_{i-1}(s) \Delta s .
\end{gathered}
$$

Theorem 1. Assume that (A1)-(A2) and (4). If the equation

$$
I_{n-1}^{\beta}(\delta(v)) Q(v) y^{\frac{\beta}{\alpha}}(\delta(v))+y^{\Delta}(v)=0,
$$

is oscillatory, where $Q$ is defined as in Lemma 6 . Then equation (1) is oscillatory.

Proof. If $x$ is a non-oscillatory solution of (1). $x(v)>0, x(\delta(v))>0, x(\tau(v))>0, v \in\left[v_{1}, \infty\right)_{T}$, $\left(v_{1} \geq v_{0}\right)$. From Lemma 6, we have (5) and (8). Letting $\left(z^{\Delta^{n-1}}(v)\right)^{\alpha} r(v)=: y(v)$, then

$$
\left(\frac{y(v)}{r(v)}\right)^{\frac{1}{\alpha}}=z^{\Delta^{n-1}}(v) .
$$

We know that $y^{\Delta}(v)<0,\left[v_{1}, \infty\right)_{T}$, then integrating (13), we can get

$$
\int_{v_{1}}^{v} r^{-\frac{1}{\alpha}}(s) \Delta s \times y^{\frac{1}{\alpha}}(v) \leq z^{\Delta^{n-2}}(v),
$$

i.e.

$\left(A(v)-A\left(v_{1}\right)\right) y^{\frac{1}{\alpha}}(v)=I_{1}(v) y^{\frac{1}{\alpha}}(v) \leq z^{\Delta^{n-2}}(v)$. 
Integrating (14) $(n-2)$ times from $v_{1}$ to $v$, we have

$$
I_{n-1}(v) y^{\frac{1}{\alpha}}(v) \leq z(v)
$$

then

$$
I_{n-1}(\delta(v)) y^{\frac{1}{\alpha}}(\delta(v)) \leq z(\delta(v)) .
$$

Combining (15) together with (8), and we get $y(v)>0$ is a solution of

$$
I_{n-1}^{\beta}(\delta(v)) Q(v) y^{\frac{\beta}{\alpha}}(\delta(v))+y^{\Delta}(v) \leq 0 .
$$

Thus, from [6], (12) admits a non-oscillatory solution, too. This contradiction completes the proof.

Theorem 2. Let $n>2, \beta \geq \alpha$. Assume that (A1)(A2) and (4). If there exist $\phi \in C_{r d}^{1}\left(\left[v_{0}, \infty\right)_{T}, R\right)$ for $M>0, v_{1} \in\left[v_{0}, \infty\right)_{T}$ we have

$$
\begin{aligned}
& \limsup \int_{v_{2}}^{v}\left[Q ( u ) \phi ( u ) \left(\int _ { v _ { 1 } } ^ { \rho ^ { n - 3 } ( \delta ( u ) ) } \left(h_{n-3}(\delta(u), \sigma(\eta))\right.\right.\right. \\
& \left.\left.\int_{v_{1}}^{\eta} r^{-1 / \alpha}(s) \Delta s\right) \Delta \eta\left(\int_{v_{1}}^{u} \frac{1}{r^{l \alpha}(s)} \Delta s\right)^{-1}\right)^{\beta} \\
& \left.-r(u) \alpha^{\alpha}\left(\left(\phi^{\Delta}(u)\right)_{+}\right)^{\alpha+1}\left((\beta M)^{\alpha}(\alpha+1)^{\alpha+1} \phi^{\alpha}(u)\right)^{-1}\right] \Delta u=\infty
\end{aligned}
$$

where $v_{2} \in\left[v_{1}, \infty\right)_{T}, \rho^{n-3}(\delta(v)) \geq v_{1}$ for $v \in\left[v_{2}, \infty\right)_{T}, Q(u)$ is defined as in Lemma $6,\left(\phi^{\Delta}(u)\right)_{+}=\max \left\{\phi^{\Delta}(u), 0\right\}$. Then, (1) is oscillatory.

Proof. If $x$ is a non-oscillatory solution of (1). $\quad x(v)>0, \quad x(\tau(v))>0, \quad x(\delta(v))>0$, $v \in\left[v_{1}, \infty\right)_{T},\left(v_{1} \geq v_{0}\right)$. By Lemma 6 , we have (5) and (8). Define a generalized Ricati substitution

$$
\omega(v)=\phi(v)\left(z^{\Delta^{n-1}}(v)\right)^{\alpha}\left(z^{\Delta^{n-2}}(v)\right)^{-\beta} r(v), v \in\left[v_{1}, \infty\right)_{T} .
$$

Obviously, $\omega(v)>0$. Then

$$
\begin{aligned}
& \omega^{\Delta}(v) \\
= & \left(z^{\Delta^{n-2}}(v)\right)^{-\beta} \phi(t)\left(\left(z^{\Delta^{n-1}}(v)\right)^{\alpha} r(v)\right)^{\Delta} \\
& +\left(z^{\Delta^{\Delta^{-1}}}(\sigma(v))\right)^{\alpha} r(\sigma(v))\left(\left(z^{\Delta^{n-2}}(v)\right)^{-\beta} \phi(t)\right)^{\Delta} \\
\leq & -\left(z^{\Delta^{n-2}}(v)\right)^{-\beta} Q(v) z^{\beta}(\delta(v)) \phi(v) \\
& +\left(z^{\Delta^{n-1}}(\sigma(v))\right)^{\alpha} r(\sigma(v))\left(z^{\Delta^{n-2}}(v)\right)^{\beta}\left(z^{\Delta^{n-2}}(v)\right)^{-\beta} \\
& \left(z^{\Delta^{n-2}}(\sigma(v))\right)^{-\beta} \phi^{\Delta}(v) \\
& -\left(\left(z^{\Delta^{n-2}}(v)\right)^{\beta}\right)^{\Delta}\left(z^{\Delta^{n-2}}(v)\right)^{-\beta} \phi(v)\left(z^{\Delta^{n-2}}(\sigma(v))\right)^{-\beta} \\
= & -\left(z^{\Delta^{n-2}}(v)\right)^{-\beta} Q(v) \phi(v) z^{\beta}(\delta(v)) \\
& +\phi^{-1}(\sigma(v)) \omega(\sigma(v)) \phi^{\Delta}(v) \\
& -\phi(v)\left(z^{\Delta^{n-1}}(\sigma(v))\right)^{\alpha}\left(\left(z^{\Delta^{n-2}}(v)\right)^{\beta}\right)^{\Delta} r(\sigma(v)) \\
& \left(z^{\Delta^{n-2}}(v)\right)^{-\beta}\left(z^{\Delta^{n-2}}(\sigma(v))\right)^{-\beta} .
\end{aligned}
$$

By Lemma 7,

$$
\begin{aligned}
& \left(z^{\Delta^{n-2}}(v)\right)^{-\beta} z^{\beta}(\delta(v)) \\
= & \left(z(\delta(v))\left(z^{\Delta^{n-2}}(\delta(v)) z^{\Delta^{n-2}}(v)\right)^{-1} z^{\Delta^{n-2}}(\delta(v))\right)^{\beta} \\
\geq & \left(\frac{\int_{v_{1}}^{\rho^{n-3}(\delta(v))}\left(h_{n-3}(\delta(v), \sigma(\eta)) \int_{v_{1}}^{\eta} \frac{1}{r^{\prime \alpha}(s)} \Delta s\right) \Delta \eta}{\int_{v_{1}}^{v} \frac{1}{r^{1 / \alpha}(s)} \Delta s}\right)^{\beta}
\end{aligned}
$$

where $\rho^{n-3}(\delta(v)) \geq v_{1}, \quad v \in\left[v_{2}, \infty\right)_{T} \subseteq\left[v_{1}, \infty\right)_{T}$. Moreover, by Lemma 5 , we obtain

$$
\begin{aligned}
& \left(\left(\eta^{\Delta^{n-2}}(v)\right)^{\beta}\right)^{\Delta} \\
= & \int_{0}^{1}\left[\eta^{\Delta^{n-2}}(\sigma(v)) h+\eta^{\Delta^{n-2}}(v)(1-h)\right]^{\beta-1} d h \times \eta^{\Delta^{n-1}}(v) \beta \\
\geq & \left\{\begin{array}{c}
z^{\Delta^{n-1}}(v) \beta\left(z^{\Delta^{n-2}}(v)\right)^{\beta-1} \text { if } \beta \geq 1, \\
z^{\Delta^{n-1}}(v) \beta\left(z^{\Delta^{n-2}}(\sigma(v))\right)^{\beta-1} \text { if } 0<\beta<1 .
\end{array}\right.
\end{aligned}
$$

Using (18) and (19) in (17), we have: If $\beta \geq 1$, then $\omega^{\Delta}(v)$

$$
\begin{aligned}
& \leq-\phi(v)\left(\frac{\int_{v_{1}}^{\rho^{n-3}(\delta(v))}\left(h_{n-3}(\delta(v), \sigma(\eta)) \int_{t_{1}}^{\eta} \frac{1}{r^{\nu / \alpha}(s)} \Delta s\right) \Delta \eta}{\int_{v_{1}}^{v} \frac{1}{r^{\prime \alpha}(s)} \Delta s}\right)^{\beta} Q(v) \\
& +\phi^{-1}(\sigma(v)) \omega(\sigma(v)) \phi^{\Delta}(v) \\
& \quad-\beta \phi(v)\left(z^{\Lambda^{\nu^{-1}}}(\sigma(v))\right)^{\alpha} z^{\Delta^{n-2}}(\sigma(v)) r(\sigma(v)) \\
& \quad\left(z^{\Delta^{n-2}}(v)\right)^{-1} z^{\Delta^{n-1}}(v)\left(z^{\Delta^{n-2}}(\sigma(v))\right)^{-\beta-1} \\
& \quad \text { If } 0<\beta<1, \text { then }
\end{aligned}
$$

$\omega^{\Delta}(v)$

$$
\begin{aligned}
\leq & -\phi(v)\left(\int_{v_{1}}^{\rho^{n-3}(\delta(v))}\left(h_{n-3}(\delta(v), \sigma(\eta)) \int_{v_{1}}^{\eta} \frac{1}{r^{\gamma / \alpha}(s)} \Delta s\right) \Delta \eta\left(\int_{v_{1}}^{v} \frac{1}{r^{\alpha / \alpha}(s)} \Delta s\right)^{-1}\right)^{\beta} \\
& Q(v)+\frac{\phi^{\Delta}(v)}{\phi(\sigma(v))} \omega(\sigma(v)) \\
& -\left(z^{\Delta^{n-2}}(\sigma(v))\right)^{-\beta-1} \phi(v)\left(z^{\Delta^{n-1}}(\sigma(v))\right)^{\alpha} \beta z^{\Delta^{n-1}}(v) \\
& \left(z^{\Delta^{n-2}}(v)\right)^{-\beta} r(\sigma(v))\left(z^{\Delta^{n-2}}(\sigma(v))\right)^{\beta} .
\end{aligned}
$$

Since $z^{\Delta^{n-1}}(v)>0$ and $\left(\left(z^{\Delta^{n-1}}(v)\right)^{\alpha} r(v)\right)^{\Delta}<0$ on $\left[v_{1}, \infty\right)_{T}$,

$$
\begin{gathered}
z^{\Delta^{n-2}}(\sigma(v)) \geq z^{\Delta^{n-2}}(v), \\
\left(\frac{r(\sigma(v))}{r(v)}\right) z^{\Delta^{n-1}}(\sigma(v)) \leq z^{\Delta^{n-1}}(v), v \in\left[v_{1}, \infty\right)_{T} .
\end{gathered}
$$

So, (20), (21) yield 


$$
\begin{aligned}
& \omega^{\Delta}(v) \\
\leq & -\phi(v)\left(\frac{\int_{t_{1}}^{\rho^{n-3}(\delta(v))}\left(h_{n-3}(\delta(v), \sigma(\eta)) \int_{t_{1}}^{\eta} \frac{1}{r^{/ \alpha}(s)} \Delta s\right) \Delta \eta}{\int_{v_{1}}^{v} \frac{1}{r^{1 / \alpha}(s)} \Delta s}\right)^{\beta} \\
& Q(v)+\phi^{-1}(\sigma(v)) \omega(\sigma(v)) \phi^{\Delta}(v) \\
& -\left(z^{\Delta^{n-1}}(\sigma(v))\right)^{\alpha+1} \beta\left(z^{\Delta^{n-2}}(\sigma(v))\right)^{-\beta-1} \phi(v) \\
& r^{-1 / \alpha}(v) r^{(\alpha+1) / \alpha}(\sigma(v))
\end{aligned}
$$

Then

$-r(\sigma(v)) \phi(v)\left(z^{\Delta^{n-2}}(\sigma(v))\right)^{-\beta-1} \beta\left(z^{\Delta^{n-1}}(\sigma(v))\right)^{\alpha+1}=$

$-\frac{\beta \phi(v)}{\phi^{(1+\alpha) / \alpha}(\sigma(v)) r^{1 / \alpha}(v)}\left(z^{\Delta^{n-2}}(\sigma(v))\right)^{\frac{\beta-\alpha}{\alpha}} \omega^{\frac{1+\alpha}{\alpha}}(\sigma(v))$

From (5), then $\left(z^{\Delta^{n-2}}(v)\right)^{\Delta}>0,\left[v_{1}, \infty\right)_{T}$, and $\beta \geq \alpha$, then exist a constant $M>0$ such that $\left(z^{\Delta^{n-2}}(\sigma(v))\right)^{\frac{\beta-\alpha}{\alpha}} \geq M$ on $\left[v_{1}, \infty\right)_{T}$, hence

$$
\begin{aligned}
& -r(\sigma(v)) \phi(v)\left(z^{\Delta^{n-2}}(\sigma(v))\right)^{-\beta-1} \beta\left(z^{\Delta^{n-1}}(\sigma(v))\right)^{\alpha+1} \\
& \leq-\phi^{-(1+\alpha) / \alpha}(\sigma(v)) r^{-1 / \alpha}(v) \beta M \phi(v) \omega^{\frac{1+\alpha}{\alpha}}(\sigma(v)) .
\end{aligned}
$$

Using this in (22), we have

$$
\omega^{\Delta}(v)
$$$$
-\phi(v)\left(\frac{\int_{v_{1}}^{\rho^{n-3}(\delta(v))}\left(h_{n-3}(\delta(v), \sigma(\eta)) \int_{t_{1}}^{\eta} \frac{1}{r^{1 / \alpha}(s)} \Delta s\right) \Delta \eta}{\int_{v_{1}}^{v} \frac{1}{\gamma^{\prime \alpha}(s)} \Delta s}\right)^{\beta}
$$

$$
Q(v)+\phi^{-1}(\sigma(v)) \omega(\sigma(v))\left(\phi^{\Delta}(v)\right)_{+}
$$$$
-M \phi^{-(1+\alpha) / \alpha}(\sigma(v)) \beta r^{-1 / \alpha}(v) \phi(v) \omega^{\frac{1+\alpha}{\alpha}}(\sigma(v))
$$

Letting

$$
\begin{gathered}
E=\phi^{-1}(\sigma(v))\left(\phi^{\Delta}(v)\right)_{+}, \\
G=\frac{\beta M \phi(v)}{\phi^{(1+\alpha) / \alpha}(v) r^{1 / \alpha}(\sigma(v))}
\end{gathered}
$$

and $w=\omega(\sigma(v))$. Applying the inequality

$$
E w-G w^{\frac{\alpha+1}{\alpha}} \leq \frac{\alpha^{\alpha}}{(\alpha+1)^{\alpha+1}} \frac{E^{\alpha+1}}{G^{\alpha}}, G>0,
$$

we conclude from (23) that

$$
\omega^{\Delta}(v) \leq-Q(v) \phi(v)\left(\frac{\int_{v_{1}}^{\rho^{n-3}(\delta(v))}\left(h_{n-3}(\delta(v), \sigma(\eta)) \int_{v_{1}}^{\eta} \frac{1}{r^{\prime \mu \alpha}(s)} \Delta s\right) \Delta \eta}{\int_{v_{1}, \frac{1}{\nu \alpha}(s)}^{v} \Delta s}\right)^{\beta}
$$

$$
\begin{aligned}
& \int_{v_{2}}^{v}[Q(u) \phi(u) \\
& \left(\frac{\int_{v_{1}}^{\rho^{n-3}}(\delta(u))}{\left(h_{n-3}(\delta(u), \sigma(\eta)) \int_{v_{1}}^{\eta} \frac{1}{r^{1 / \alpha}(s)} \Delta s\right) \Delta \eta}\right)^{\beta} \\
& \int_{v_{1}}^{u} \frac{1}{r^{1 / \alpha}(s)} \Delta s \\
& \left.-\frac{\alpha^{\alpha} r(u)\left(\left(\phi^{\Delta}(u)\right)_{+}\right)^{\alpha+1}}{(\alpha+1)^{\alpha+1}(\beta M)^{\alpha} \phi^{\alpha}(u)}\right] \Delta u \leq \omega\left(v_{2}\right),
\end{aligned}
$$

which contradicts (16). This complete the pro0f.

\section{Conclusion}

In this paper, applying new technique, some criteria for (1), include comparison theorem and Phil0s-type oscillation criteria. Our result obtained are new even for the case $T=R$ and $T=Z$.

\section{Acknowledgements}

This study was funded by Shadong Provincial Science Foundation (ZR2016AM17, ZR2017MA043), and the Natural Foundation of China (617043180).

\section{References}

1. S. R. Grace, On the oscillation of higher order dynamic equations, J. Adv. Res. DOI.org/10.1016/j.jare.2012.04.003

2. E. Tumç, Oscillation results for even order functional dynamic equations, Electron. J. Qual. Theory Differ. Equ. DOI.org/10.1.1.642.5480.

3. S. Hilgar, Analysis on measure chains - a unified approach to continuous and discrete calculus, Results Math. https:// link.springer. com/article/10.1007/BF03323153.

4. T. Li, Z. Han, C. Zhang, S. Sun, On the oscillation of second-order Emdn-Fowlr neutral differential equations, J. Apl. Math. Compt. 317 (2011) 601610.

5. M. Bohmer, A. Peterson, Advances in Dynamic Equations on Time Scales, Birkhäser, ISBN0-81764225-0.

6. R. P. Agrwal, M. Boher, Basic calculus and some of its applications, Results Math. https:// link.springer.com/article/10.1007/BF03322019.

7. Q. Ha0, F. Lu, Oscillation theorem for superliner second order damped differential equations, Apl. Math. Compt, 217 (2011) 7126-7131.

8. Y. Bolt, Oscillation criteria for nonlinear secondorder difference equations with a nonlinear damped term, Apl. math. Let. 18 (2005) 309-338.

9. J. Jia, X. Li, Oscillation of second order nonlinear neutral differential equations, Apl. Math. Compt, 135 (2003) 531-540.

10. Y. Sui, and S. Sun, Oscillation of third order dynamic equation with mixed arguments on time scales, Adv. Diff. Eq., DOI. org/10.1186/s 1366 018-1654-3. 
11. Y. Sui, Z. Han, Oscillation of third-order nonlinear dynamic equation with damping term, J. Apl. Math.
Compt. (2018) 58, DOI. org/10.1007/s12190-0171158-4. 